\title{
Analyzing the trade-off between training session time and performance in myoelectric hand gesture recognition during upper limb movement
}

\author{
Matteo Cognolato*1,2, Lorenzo Brigato*3 ${ }^{* 3}$ Yashin Dicente Cid $^{1}$, Manfredo Atzori ${ }^{1}$ and Henning Müller ${ }^{1,4}$
}

\begin{abstract}
Although remarkable improvements have been made, the natural control of hand prostheses in everyday life is still challenging. Changes in limb position can considerably affect the robustness of pattern recognition-based myoelectric control systems, even if various strategies were proposed to mitigate this effect. In this paper, we investigate the possibility of selecting a set of training movements that is robust to limb position change, performing a trade-off between training time and accuracy. Four able-bodied subjects were recorded while following a training protocol for myoelectric hand prostheses control. The protocol is composed of 210 combinations of arm positions, forearm orientations, wrist orientations and hand grasps. To the best of our knowledge, it is among the most complete including changes in limb positions. A training reduction paradigm was used to select subsets of training movements from a group of subjects that were tested on the left-out subject's data. The results show that a reduced training set (30 to 50 movements) allows a substantial reduction of the training time while maintaining reasonable performance, and that the trade-off between performance and training time appears to depend on the chosen classifier. Although further improvements can be made, the results show that properly selected training sets can be a viable strategy to reduce the training time while maximizing the performance of the classifier against variations in limb position.
\end{abstract}

\section{INTRODUCTION}

In recent years, remarkable advances have been made in the development of pattern recognition methods to control dexterous robotic hand prostheses. Pattern recognition-based myocontrol systems are now widely studied and applied in scientific research. Despite the many advantages with respect to the classic myocontrol strategy, the results are still poorly translated into clinical applications and commercial products [1], [2], [3]. This gap is due to several aspects, particularly the numerous factors potentially influencing the Surface Electromyography (sEMG) signals, preventing the pattern recognition approach to reach the desired robustness in unconstrained settings [3], [2], [4]. In particular, changes in limb position were shown to influence the task of classifying hand gestures for the control of hand prostheses considerably [3], [5]. The influence that the variations of limb position has on the accuracy of decoding the subject's

\footnotetext{
*M. Cognolato and L. Brigato are co-first authors.

${ }^{1}$ M. Cognolato, Y. Dicente Cid, M. Atzori and H. Müller are with the Information Systems Institute, University of Applied Sciences Western Switzerland (HES-SO Valais), Sierre, Switzerland. matteo.cognolatolhevs.ch

${ }^{2} \mathrm{M}$. Cognolato is with the Rehabilitation Engineering Laboratory, Department of Health Sciences and Technology, ETH Zurich, Zurich, Switzerland.

${ }^{3} \mathrm{~L}$. Brigato is with the Department of Computer, Control and Management Engineering, Sapienza University of Rome, Rome, Italy.

${ }^{4} \mathrm{H}$. Müller is with the University of Geneva, Geneva, Switzerland.
}

movement intent with the use of pattern recognition methods was investigated and reported in several studies e.g. [5], [6], [7], [8], [9].

Fougner et al. [8] studied the influence of variations in limb postures on eight motion classes performed in $5 \mathrm{limb}$ positions, using the well-known Time Domain (TD) [10] features and the Linear Discriminat Analysis (LDA) classifier. The results showed that the average classification error decreases from $18 \%$ to $5.7 \%$ by training the classifier in multiple limb positions and a further improvement of $0.7 \%$ was obtained including accelerometry data. Liu et al. [11] monitored the effects of dynamic changes on patterns with three metrics regarding a hyperellipsoidal representation of feature vectors. The effect of arm movements on the recognition rate of hand and wrist motions are reflected in the changes of sEMG pattern characteristics from one arm position to another and ranged from an average of $5.26 \%$ (intra-condition) to $20.98 \%$ (inter-condition) in terms of classification accuracy. Betthauser et al. [12] investigated the use of a classifier that is less sensitive to signal variations resulting from untrained limb positions using a sparse Extreme Learning Machine (ELM) with Adaptive Sparse Representation Classification (EASRC) algorithm. The classification accuracy as a function of train-test distance for two amputee subjects wearing a prosthesis was chosen as a metric to judge the classifier performance. The proposed algorithm showed a good classification accuracy as function of train-test distance in both off-line and on-line tests with transradial and ablebodied subjects. Radman et al. [13] investigated the level of robustness that various feature sets and classifiers provide when classifying data from dynamic limb movements, with a focus on the commonly used TD features and LDA. They showed that LDA provides good performance when classifying dynamic movements and the classification accuracy can be further improved by including or replacing some of the standard TD feature components. Miselovic et al. [14] explored the effects of postural and temporal variability in the recognition of hand gestures via myoelectric signals. They acquired and released a data set including six grasp-types recorded in four arm positions and repeated for eight days, showing that the Support Vector Machine (SVM) with radial basis function (RBF) kernel provides the highest accuracy at the cost of higher computational requirements, whereas LDA was the most robust model for inter-posture grasp recognition with the lowest computational needs. Jiang et al. [9] investigated the influence of arm position on the performance of a simultaneous and proportional control approach. Their results confirmed that changes in arm position negatively impact the 
performance of the algorithm, showing that this influence is less pronounced in amputees.

Other improvements in this respect were made on the features describing the sEMG signal. Several features have been proposed and evaluated over the years, showing diverse performance [15]. Novel features designed to be more robust against changes in limb position, forearm orientation and force level variation have been proposed [16], [17], [18], [19]. In particular, a new set of features called fused TimeDomain Descriptors (fTDD) were presented in [19]. These features are an extension of the spectral-moments-based features introduced in [17], that were shown to be more robust for pattern recognition-based hand gesture classification in presence of limb position variations [17], [18].

Despite several advances, the adversarial effect of upper limb movements in pattern recognition-based myoelectric control still represents a challenging task. One of the reasons may be that, although dynamic movements with variations in arm position, forearm orientation, wrist orientation and hand grasp are common in daily life activities, movements simultaneously involving more than one or two joints are not often investigated. One of the most effective solutions to increase the robustness of the classifier against limb movements is to train it in multiple limb positions [5], [8]. However, this can lead to long and tiring training sessions, making it unfeasible for real-life applications [8], [9].

In this work, we aim at exploring and evaluating whether it is possible to select a training set reducing the training time while maintaining comparable performance in presence of limb position variations. In order to do so, we first investigate the performance of the TD, marginal Discrete Wavelet Transform (mDWT) [20] and fTDD features in classifying hand gestures during dynamic and complex upper limb movements. Then, we explore the variability of the classification accuracy as a function of the training set dimension and, finally, we investigate the feasibility of the approach by selecting a subset of movements from a pool of subjects that can be used as training set for a new subject.

\section{METHODS}

This section includes the description of the acquisition setup, the acquisition protocol, the pre-processing procedure and the mathematical description of the inter-subject training set reduction paradigm.

\section{A. Acquisition setup}

sEMG and accelerometry data were recorded at $1925.9 \mathrm{~Hz}$ and $148.1 \mathrm{~Hz}$ respectively using a Trigno Lab Wireless system (Delsys Inc., USA). Eight electrodes were equally spaced on the subject's forearm at the height of the radiohumeral joint. A second array composed of four electrodes was placed next to the first one, in a more distal position. A single electrode was positioned at the wrist level to record accelerometry data (Fig. 1). A custom-made software was used to record the data and to guide the subject through the exercise. Vocal instructions synthesized with a text-to-speech engine were used to interact with the subject. A total of 4 able-bodied participants ( 3 males and 1 female; age $29.3 \pm 6.2$ years old, height $177.0 \pm 6.8 \mathrm{~cm}$ ) participated in the study.

\section{B. Experimental protocol}

A set of movements was obtained starting from the postures reported in Tab. II. Each movement was defined as a combination of postures of the different segments of the upper limb, including the upper arm, the forearm, the wrist and the hand e.g. performing a lateral grasp with arm forward $\left(90^{\circ}\right)$, forearm pronated, and wrist in radial deviation. Three grasp-types were chosen according to their importance for activities of daily living (medium wrap, lateral and precision disk), together with a no grasp hand posture. All the possible movements were tested by two able-bodied subjects in order to evaluate their feasibility and intuitiveness. This evaluation allowed to select the movements that a person would intuitively perform in everyday life conditions. After this process, a total of 210 movements were chosen. In order to make

TABLE I

THE LIST OF POSTURES THAT WERE COMBINED TO FORM A MOVEMENT.

\begin{tabular}{cccc} 
Arm & Forearm & Wrist & Hand \\
\hline forward & & neutral & no grasp \\
upward & neutral & flexion & medium wrap \\
downward & supinated & extension & lateral \\
internal & pronated & $\begin{array}{c}\text { radial deviation } \\
\text { ulnar deviation }\end{array}$ & precision disk \\
external & & carion
\end{tabular}

the data recording easily reproducible, the acquisitions were performed using a bookshelf and real objects. The position of the objects was defined in accordance with the movements and it was kept as similar as possible for the three grasptypes, as depicted in Fig. 1. A set of identical objects for each grasp type were attached to a wooden tray to guarantee the same placement for each subject. In addition, the height of the objects on the bookshelves was further adjusted according to the subject's height, maintaining a shoulder flexion angle of $\sim 45^{\circ}$ for the downward position, $\sim 90^{\circ}$ for the forward position and $\sim 135^{\circ}$ for the upward position. The subjects were asked to stand in front of the bookshelf
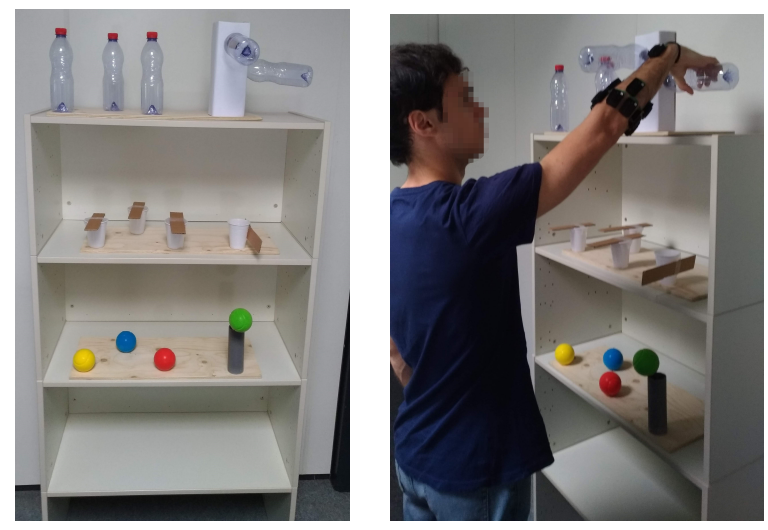

Fig. 1. The bookshelf and the objects used in the experiment. Sets of empty 0.51 plastic bottles, cardboard stripes and sponge balls were used for the medium wrap, lateral and precision disk grasps respectively.

and were instructed to execute the movements as naturally 
as possible. Each movement was performed dynamically, starting from a neutral position with the arm at the side of the body. The arm position, forearm orientation, wrist orientation as well as grasp-type had to be adjusted simultaneously to perform the requested movement. The subjects were guided by the acquisition software via vocal instructions, providing both the description of the movement to perform (taking on average $4 s$ ), and indicating the beginning of the movement and when the subject should return to the rest position. Each trial was composed of $3 s$ of movement and $3 s$ of resting, repeated three times. Therefore, the total time for the pure signal recording was approximately of 105 minutes. Initial technical preparations were usually thirty minutes long and three pauses of roughly five minutes each were included in order to limit muscular and mental fatigue. The length of a complete acquisition session approximately reached two hours and a half. The acquisition protocol was approved by the Commission Cantonale Valaisanne d'étique médicale.

\section{Data pre-processing and feature extraction}

The raw sEMG and accelerometry data were first preprocessed. This step checks the consistency of the data and removes the power line interference at $50 \mathrm{~Hz}$. Then, three signal features, namely TD, mDWT, and fTDD [21], were extracted using a sliding window approach (window length of $200 \mathrm{~ms}$ with an overlap of $20 \mathrm{~ms}$ ) [10]. Based on previous results, we decided to use the $7^{\text {th }}$ order Daubechies wavelet function considering the marginal coefficients up to the third level [22], [20]. Due to a technical failure, the data from two electrodes of the distal array were not recorded. The analysis was therefore performed using only the 8 proximal electrodes for all the subjects in order to obtain comparable results. Only the sEMG data were considered in the analysis.

\section{Variability analysis}

A random sampling was applied to evaluate the variability of the classification accuracy as the size of the training set dimension changes. More precisely, 200 random assignments were performed for each dimension, from $B=1$ to $B=$ 209 (with $B$ being the cardinality of the selected subset of movements), to construct a probability distribution. Formally, given the random variable $A_{B}$ as the accuracy scored by a training set of dimension $B$, we approximate all the possible outcomes with a Gaussian distribution $a_{B}$ characterized by its mean $\mu_{a_{B}}$ and standard deviation $\sigma_{a_{B}}$ computed over the 200 random samples. The analysis was performed only with the fTDD features using the LDA classifier.

\section{E. Training set reduction method}

An algorithm was tested to select target movements using an incremental average ranking based on predictive model scores (Incremental Average Algorithm (IAA)). Since the IAA evaluates a model at each iteration, the chosen classifier was LDA due to its low computational requirements. The algorithm follows an $\epsilon-$ greedy policy to select the movements. In other words, the greedy paradigm follows the best local option (the movements) with probability $1-\epsilon$, and a random one with probability $\epsilon$. Preliminary experiments on our data revealed that the best value for $\epsilon$ was 0.1 . The algorithm selects a pool of a predefined dimension $B$ at each iteration. Afterwards, the LDA model is trained with the chosen $B$ movements and validated on a different repetition of the overall training set (210 movements). The validation consisted in the classification of the four grasp-types. This process outputs a validation accuracy $\alpha^{(t)}$ that is the value needed to update the performance metric $\theta^{(t)}$ in $\theta^{(t+1)}$, namely the average accuracy scored by each movement $m$ at iteration $t+1$ :

$$
\theta_{m}^{(t+1)}=\theta_{m}^{(t)}+\frac{1}{N_{m}^{(t)}}\left(\alpha^{(t)}-\theta_{m}^{(t)}\right)
$$

with $N_{m}^{(t)}$ being the number of times the movement $m$ is selected. The algorithm outputs the labels of the movements that scored the highest average accuracy in the validation step. As a shorter training time is preferable and the IAA has a high computational cost, we focused the analysis on subsets of the training set requiring fewer than 30 minutes to be recorded, corresponding to $B \in\{10,20,30,40,50\}$ movements. Three other evaluations were done for $B \in$ $\{75,110,150\}$ to explore the overall trend, resulting in 8 training sets composed of $B$ movements selected by the IAA. The training reduction paradigm is repeated three times to explore the variability of the algorithm. In our experiments we set the number of iterations to $5 \times 10^{4}$.

\section{F. Inter-subject training set evaluation}

The grasp type classification is executed by training and testing with a linear and a non-linear classifier, namely LDA and Random Forest (RF). The investigation was performed using an inter-subject training set reduction approach. In other words, the first step consisted of the selection of subsets composed of $B$ training movements from the data of the $S-1$ subjects using the IAA. Then, the training set for the $S^{t h}$ subject was created using the same types of movements that were selected. While the IAA only selected the subset of $B$ movements, both training and testing were performed using the data of the new subject $S^{\text {th }}$.

In both variability analysis and inter-subject training set evaluation the classifiers were trained with two repetitions (the ones used to train and validate the IAA) and the testing set was created using the remaining repetition, which was unknown to the models. The classification was performed on the grasp-type, having a 4-class problem (no grasp, medium wrap, lateral and precision disk). The data set was slightly unbalanced ( 75 movements in the no grasp class and 45 in the others), with a baseline accuracy of $75 / 210=0.3571$.

\section{RESULTS}

First, we evaluated the performance of the hand gesture classification on the proposed protocol, which includes 210 movements with dynamic changes in arm position, forearm orientation, wrist orientation and hand grasp. The performance was evaluated using the inter-subject training set reduction approach described in the previous section, 
with the TD, mDWT, fTDD features and the LDA and RF classifiers. The results are reported in Tab. II] where the size of the training set is referred to as $B$ and the results are reported as average classification accuracy and standard deviation among all the subjects and for both LDA and RF. On the leftmost column of Tab. II we show the length of the pure signal acquisition time with $B$ movements.

The maximum average classification accuracy for the four grasp-types is $85.89 \%$. This value is obtained with RF trained on all 210 movements and the fTDD features. The same features achieved the highest performance also with LDA, with a maximum average of $78.81 \%$. The fTDD consistently exhibited best performance in terms of average classification accuracy, therefore only this feature type was considered for the subsequent analyses.

TABLE II

INTER-SUBJECT TRAINING SET ACCURACY (\%) WITH TD, MDWT AND FTDD FEATURES USING THE LDA AND RF CLASSIFIERS.

\begin{tabular}{|c|c|c|c|c|c|}
\hline & $\begin{array}{l}\text { Time } \\
{[\mathrm{min}]}\end{array}$ & B & TD & mDWT & fTDD \\
\hline \multirow{9}{*}{ 氐 } & 5 & 10 & $51.13 \pm 8.74$ & $52.75 \pm 7.17$ & $61.05 \pm 7.01$ \\
\hline & 10 & 20 & $54.09 \pm 5.32$ & $54.92 \pm 5.75$ & $68.06 \pm 5.75$ \\
\hline & 15 & 30 & $54.07 \pm 7.15$ & $55.67 \pm 5.52$ & $68.99 \pm 6.07$ \\
\hline & 20 & 40 & $57.53 \pm 9.14$ & $55.85 \pm 6.47$ & $72.24 \pm 5.78$ \\
\hline & 25 & 50 & $59.89 \pm 7.87$ & $58.08 \pm 5.09$ & $74.53 \pm 4.96$ \\
\hline & 38 & 75 & $63.14 \pm 7.36$ & $59.41 \pm 5.06$ & $74.97 \pm 4.63$ \\
\hline & 55 & 110 & $63.22 \pm 7.84$ & $59.17 \pm 4.55$ & $77.54 \pm 3.23$ \\
\hline & 75 & 150 & $65.30 \pm 8.07$ & $59.24 \pm 5.37$ & $78.33 \pm 4.32$ \\
\hline & 105 & 210 & $65.54 \pm 7.57$ & $60.47 \pm 5.17$ & $78.81 \pm 4.16$ \\
\hline \multirow{9}{*}{$\frac{1}{\alpha}$} & 5 & 10 & $52.18 \pm 11.31$ & $52.20 \pm 10.10$ & $53.75 \pm 8.58$ \\
\hline & 10 & 20 & $58.13 \pm 7.45$ & $62.76 \pm 8.44$ & $66.73 \pm 7.38$ \\
\hline & 15 & 30 & $61.50 \pm 7.82$ & $65.46 \pm 8.53$ & $69.21 \pm 7.77$ \\
\hline & 20 & 40 & $65.40 \pm 8.12$ & $67.49 \pm 9.34$ & $73.43 \pm 7.27$ \\
\hline & 25 & 50 & $68.73 \pm 8.28$ & $71.29 \pm 6.81$ & $75.13 \pm 7.67$ \\
\hline & 38 & 75 & $73.36 \pm 7.25$ & $75.58 \pm 5.54$ & $78.95 \pm 5.28$ \\
\hline & 55 & 110 & $78.48 \pm 7.11$ & $79.10 \pm 7.05$ & $82.76 \pm 4.37$ \\
\hline & 75 & 150 & $81.59 \pm 6.97$ & $82.12 \pm 5.90$ & $85.84 \pm 5.39$ \\
\hline & 105 & 210 & $84.92 \pm 5.74$ & $85.07 \pm 5.35$ & $85.89 \pm 4.60$ \\
\hline
\end{tabular}

The classification performance in randomly selected subsets of the training set (previously introduced in Sec. II-D) shows a rapid increase of the classification accuracy (roughly until $B=50$ ) and then saturates (as shown in Fig. 2). The detailed results reported in the $a_{B}$ column of Tab. III show that the variability decreases as the training set dimension increases, being already lower than $\pm 2 \%$ when training with $B=50$ movements. Therefore, we focused the investigation on sets of 10 and 50 movements to explore the characteristics that a training set should have to assure a high ratio between performance and training time.

The results of the inter-subject training set evaluation analysis (as described in Sec. II-F) are reported in Fig. 3. The average classification accuracy ranges between $54.31 \%$ and $84.51 \%$ for LDA and between $50.15 \%$ and $92.45 \%$ for RF. The gaps between the classification accuracy obtained with the whole training set of 210 movements and the one achieved with $B=50$ movements are, on average, of $4.65 \%$ for LDA and $10.76 \%$ for RF. The selection of a proper training set composed of 30 - 50 movements with the fTDD and LDA allows the recording of the training data in 15 - 25 minutes (from the original 105 minutes) while
TABLE III

AVERAge ACCURACY (\%) WITH IAA AND OF $a_{B}$ USING LDA. The FULL TRAINING SET ACCURACY $(B=210)$ IS INDICATED IN BRACKETS.

\begin{tabular}{cccc}
$\mathbf{B}$ & $\boldsymbol{a}_{\boldsymbol{B}}$ & IAA & Subject \\
\hline 10 & $47.15 \pm 5.44$ & $54.31 \pm 2.13$ & \\
20 & $57.69 \pm 4.21$ & $61.27 \pm 4.30$ & 1 \\
30 & $63.32 \pm 3.05$ & $68.64 \pm 2.49$ & $(77.55 \%)$ \\
40 & $66.53 \pm 2.30$ & $68.77 \pm 1.48$ & \\
50 & $68.90 \pm 1.98$ & $71.18 \pm 1.01$ & \\
\hline 10 & $52.42 \pm 6.17$ & $62.76 \pm 3.08$ & \\
20 & $62.78 \pm 3.82$ & $67.37 \pm 1.51$ & 2 \\
30 & $68.14 \pm 2.74$ & $70.58 \pm 2.49$ & $(78.58 \%)$ \\
40 & $71.15 \pm 2.09$ & $72.81 \pm 0.90$ & \\
50 & $72.97 \pm 1.61$ & $74.09 \pm 2.09$ & \\
\hline 10 & $48.63 \pm 5.14$ & $57.78 \pm 0.91$ & \\
20 & $58.37 \pm 3.71$ & $61.41 \pm 2.15$ & 3 \\
30 & $62.98 \pm 2.82$ & $64.27 \pm 0.84$ & $(74.58 \%)$ \\
40 & $65.84 \pm 2.22$ & $67.69 \pm 1.74$ & \\
50 & $67.62 \pm 1.90$ & $69.71 \pm 0.36$ & \\
\hline 10 & $63.31 \pm 6.65$ & $69.37 \pm 1.79$ & \\
20 & $72.20 \pm 3.31$ & $77.17 \pm 2.03$ & 4 \\
30 & $76.26 \pm 2.58$ & $77.98 \pm 1.16$ & $(84.51 \%)$ \\
40 & $78.46 \pm 2.05$ & $80.27 \pm 0.31$ & \\
50 & $79.70 \pm 1.83$ & $81.64 \pm 1.08$ & \\
\hline
\end{tabular}

losing on average $8.44-4.65 \%$ of accuracy with respect to the one obtained by training with the full set of 210 movements (Tab. III). The loss in accuracy is however higher when considering the RF classifier (Fig. 3). Higher values are obtained with LDA for $B \leq 50$ with respect to RF, but higher overall accuracy is reached with the latter.

In order to evaluate the effectiveness of the inter-subject training set reduction approach, a comparison with the results obtained by choosing a random subset of fixed size $B$ coming from the probability distribution $a_{B}$ was performed (Tab. III). It is important to note that a repeated random search cannot directly be executed on the new subject, as this would inevitably imply the acquisition of the whole data set and the fundamental hypothesis of choosing only $B$ movements would vanish. As reported in Tab. III] the average classification accuracy obtained with the movements selected by the IAA is always greater than $\mu_{a_{B}}$ for the movement range $B \in[10,50]$, falling outside the $75 \%$ confidence interval of the probability distributions $a_{B}$. The largest improvements were obtained where the variability is highest, particularly for $B=10$. An average increase of up to $\sim 10 \%$ with respect to the distribution mean is obtained. However, the increase in classification accuracy is already halved when considering the case of $B=20$. An example is shown in Fig. 2.

\section{DISCUSSION}

According to the results previously presented, it is possible to select inter-subject training sets that improve the tradeoff between training time and classification accuracy, even when there are variations in upper limb position. The fTDD were also shown to be more robust against limb posture changes and the high variability of the classification accuracy for smaller randomly chosen training sets suggests that noticeable improvements can be achieved by selecting a proper training set. The proposed training set selection method exhibited larger improvements where the variability 


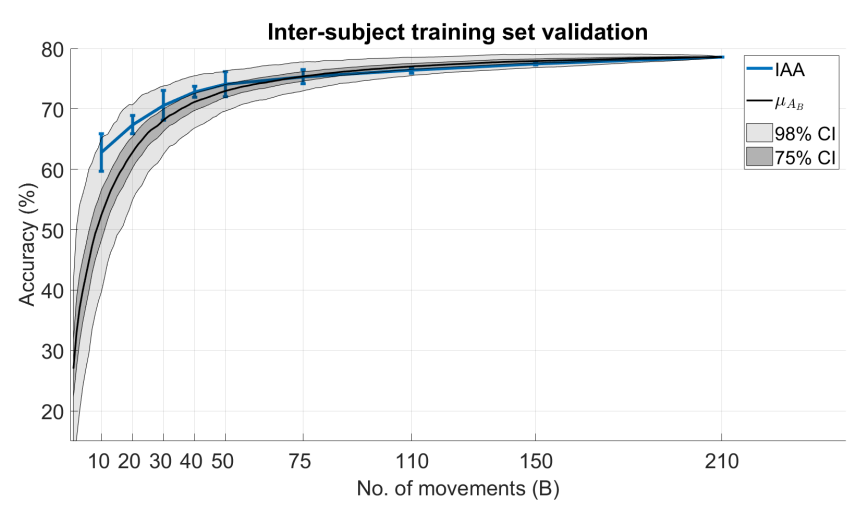

Fig. 2. This plot shows the average accuracy of the inter-subject training set evaluation (IAA for the selection phase and LDA for the testing phase) and the means and confidence intervals (referred to in the legend as $\mathrm{CI}$ ) of the probability distributions $a_{B}$ considering $B \in[1-209]$. Due to space limitation we only report the complete results belonging to subject 2 . Similar trends are obtained for the other subjects.

is higher, with an increase in classification accuracy of up to $\sim 10 \%$ with respect to the distribution mean.

The use of features that are more robust against changes in limb position can improve the robustness of myoelectric hand gesture recognition, also in presence of dynamic limb movements that are common in everyday life. It may be helpful to include such features in future studies particularly when considering different limb positions.

The variability analysis highlights how the growth in classification accuracy diminishes when the training set includes more than approximately 50 movements. Although the sample size is limited, this behaviour is consistent among all the subjects. Therefore, it does not seem worth to acquire such an extensive training set since the gain in classification accuracy is not proportional to the time needed to acquire it. Focusing on fewer than 50 movements, the variance of the probability distributions highlights the possibility that a noticeable performance improvement can be obtained by selecting a proper training set. In fact, the gap between the classification accuracy obtained by training the LDA classifier with all the movements and the one obtained at the upper confidence interval of $a_{B}$ with a set of 20 movements can drop to roughly $11 \%$ for subject 1 and to $6 \%$ for subject 4 , while the time needed to acquire the training set passes from about 105 minutes to 10 minutes.

The inter-subject training set reduction approach is able to find subsets of movements that provide a reasonable accuracy while considerably reducing the training time. The growth in classification accuracy diminishes when the training set includes more than approximately 50 movements for both classifiers. This behaviour is more pronounced for LDA, and less marked for RF, suggesting that it can depend on the type of classifier chosen. The LDA classifier exhibits better performance for $B \leq 50$, RF improves over 50 movements and the two classifiers obtain similar accuracy for 50 movements. Therefore, LDA, being a fast and simple classifier, seems a good candidate to be used for hand gesture classification in presence of limb position changes, which is
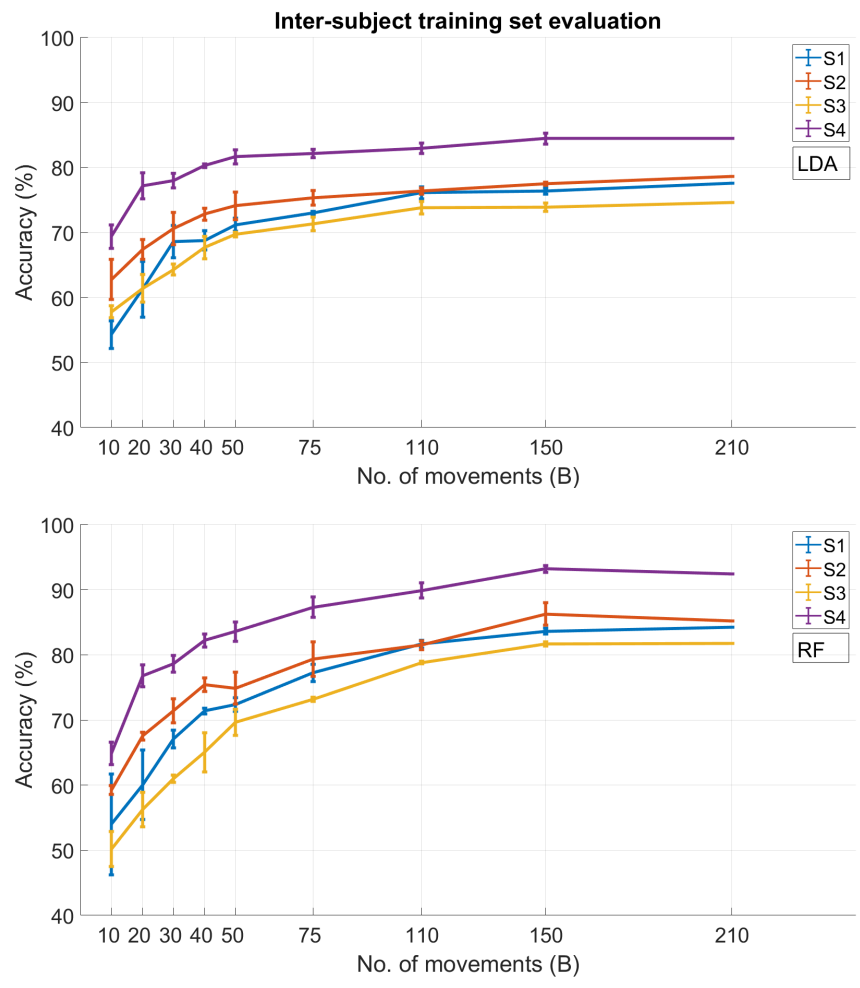

Fig. 3. Average classification accuracy for the inter-subject training set evaluation. The scores obtained for all subjects are reported. At the top, we show the accuracy obtained with LDA while at the bottom with RF. The accuracy of a single observation for $B=210$ is reported for RF.

consistent with what is found in [13], [14]. These findings seem to indicate that LDA exhibits better performance when the training set dimension is moderate. This can also be influenced by the fact that LDA was used to select the movements by the IAA and that few classes need to be classified. It was shown that better performance in terms of classification accuracy can be reached with other classifiers when a high number of classes are present [20].

The training sets selected with the IAA (where the selection is performed on more subjects and tested on the leftout one) were compared with the randomly selected sets, showing a systematic increase in classification accuracy with respect to the average random selection when the training set includes fewer than 50 movements. The comparison with acquisition protocols previously described in literature is currently in progress, however it is not straightforward due to the larger variety of movements and positions considered. Even though these values are still far from those required by a reliable interface in unconstrained environments, the results suggest the feasibility of the approach in improving the trade-off between classification accuracy and acquisition session time. Furthermore, since the anatomical differences between amputee and able-bodied subjects might be one of the reasons why arm position has a smaller influence for amputees [9], it seems plausible that this may also apply to pattern recognition approaches. It is therefore possible that the data set and consequently the training time can be 
further reduced in the actual target population. It would have been interesting to find movement subset recurrences that can be used as basic building blocks for the design of future acquisition protocols. However, all movement types seem to be necessary for the classification task. The chosen subsets are generally balanced in terms of limb, forearm, wrist and grasp categories and no clear patterns were found. Therefore, movement combinations seem to be more valuable than single movements in the model building process.

\section{CONCLUSIONS}

Reliable and robust myoelectric prosthesis control under conditions of everyday life is still challenging. Several remarkable steps have been made to improve pattern recognition-based myocontrol systems, spanning from acquisition system characteristics, new sets of features and more reliable classifiers. On the other hand, the limited robustness of the myoelectric pattern recognition techniques still limits their application in every-day life. This work tries to bring the domain one step further by increasing the robustness of such control against the adversarial influence of changes in limb position. We first defined an acquisition protocol containing dynamic movements involving variations in arm position, forearm orientation, wrist orientation and hand grasp. The protocol makes the classification task more challenging by including movements that are commonly performed in everyday life activities. Afterwards, we perform inter-subject training set reduction with the aim of maximizing the grasp-type classification accuracy while minimizing the training time. The results show that the IAA is capable of identifying a set of movements that maintain reasonable performance in terms of classification accuracy for a new subject, suggesting the feasibility of the approach. On the other hand, the improvements are limited (on average of up to $\sim 10 \%$ with respect to the average random accuracy), the highest improvements were obtained for $B=10$ where the variability is higher, and they may also be influenced by the chosen classifier. The proposed approach can also be useful in multi-posture and multi-day training strategies, in which a selected subset of movements that ensures a quick training phase can be used to re-train or update the training of the classifier daily.

Future research directions include the evaluation of the inter-subject training set selection method on a bigger sample, the investigation of other algorithms to select and classify movements and a comparison with other protocols commonly investigated in literature.

\section{ACKNOWLEDGMENT}

This work was supported by the Swiss National Science Foundation Sinergia project \#410160837 MeganePro.

\section{REFERENCES}

[1] M. Atzori and H. Mller, "Control capabilities of myoelectric robotic prostheses by hand amputees: A scientific research and market overview," Frontiers in Systems Neuroscience, vol. 9, p. 162, 2015.

[2] D. Farina and S. Amsüss, "Reflections on the present and future of upper limb prostheses," Expert Review of Medical Devices, vol. 13, no. 4, pp. 321-324, 2016.
[3] C. Castellini, P. Artemiadis, M. Wininger, A. Ajoudani, M. Alimusaj, A. Bicchi, B. Caputo, W. Craelius, S. Dosen, K. Englehart, D. Farina, A. Gijsberts, S. B. Godfrey, L. Hargrove, M. Ison, T. Kuiken, M. Marković, P. M. Pilarski, R. Rupp, and E. Scheme, "Proceedings of the first workshop on peripheral machine interfaces: Going beyond traditional surface electromyography," Frontiers in Neurorobotics, vol. 8, no. AUG, pp. 1-17, 2014.

[4] C. J. De Luca, "The Use of Surface Electromyography in Biomechanics," Journal of Applied Biomechanics, vol. 13, pp. 135-163, 1997.

[5] E. Scheme, A. Fougner, Ø. Stavdahl, A. D. Chan, and K. Englehart, "Examining the adverse effects of limb position on pattern recognition based myoelectric control," in Engineering in Medicine and Biology Society (EMBC), 2010 Annual International Conference of the IEEE. IEEE, 2010, pp. 6337-6340.

[6] K. Englehart and B. Hudgins, "A robust, real-time control scheme for multifunction myoelectric control," IEEE Trans Biomed Eng, vol. 50, no. 7, pp. 848-854, 2003.

[7] E. Scheme, K. Biron, and K. Englehart, "Improving myoelectric pattern recognition positional robustness using advanced training protocols," in Proceedings of 32nd Annual International Conference of the IEEE-EMBS. IEEE Boston, MA, 2011.

[8] A. Fougner, E. Scheme, A. D. Chan, K. Englehart, and Ø. Stavdahl, "Resolving the limb position effect in myoelectric pattern recognition," IEEE Transactions on Neural Systems and Rehabilitation Engineering, vol. 19, no. 6, pp. 644-651, 2011.

[9] N. Jiang, S. Muceli, B. Graimann, and D. Farina, "Effect of arm position on the prediction of kinematics from EMG in amputees," Medical \& Biological Engineering \& Computing, vol. 51, no. 1-2, pp. 143-151, feb 2013.

[10] B. Hudgins, P. Parker, and R. N. Scott, "A new strategy for multifunction myoelectric control," Biomedical Engineering, IEEE Transactions on, vol. 40, no. 1, pp. 82-94, 1993.

[11] J. Liu, D. Zhang, X. Sheng, and X. Zhu, "Quantification and solutions of arm movements effect on semg pattern recognition," Biomedical Signal Processing and Control, vol. 13, pp. 189-197, 2014.

[12] J. L. Betthauser, C. L. Hunt, L. E. Osborn, M. Masters, G. Lévay, R. R. Kaliki, and N. V. Thakor, "Limb position tolerant pattern recognition for myoelectric prosthesis control with adaptive sparse representations from extreme learning," IEEE Trans Biomed Eng, 2017.

[13] A. Radmand, E. Scheme, P. Kyberd, and K. Englehart, "Investigation of optimum pattern recognition methods for robust myoelectric control during dynamic limb movement," CMBES Proc., vol. 36, no. 1, 2013.

[14] B. Milosevic, E. Farella, and S. Benaui, "Exploring arm posture and temporal variability in myoelectric hand gesture recognition," in 2018 7th IEEE International Conference on Biomedical Robotics and Biomechatronics (Biorob), 2018, pp. 1032-1037.

[15] A. Phinyomark, F. Quaine, S. Charbonnier, C. Serviere, F. TarpinBernard, and Y. Laurillau, "EMG feature evaluation for improving myoelectric pattern recognition robustness," Expert Systems with Applications, vol. 40, no. 12, pp. 4832-4840, 2013.

[16] A. H. Al-Timemy, R. N. Khushaba, G. Bugmann, and J. Escudero, "Improving the Performance Against Force Variation of EMG Controlled Multifunctional Upper-Limb Prostheses for Transradial Amputees," IEEE Transactions on Neural Systems and Rehabilitation Engineering, vol. 24, no. 6, pp. 650-661, jun 2016.

[17] R. N. Khushaba, , and S. Kodagoda, "Time-dependent spectral features for limb position invariant myoelectric pattern recognition," in 2012 International Symposium on Communications and Information Technologies (ISCIT), Oct 2012, pp. 1015-1020.

[18] R. N. Khushaba, M. Takruri, J. V. Miro, and S. Kodagoda, "Towards limb position invariant myoelectric pattern recognition using timedependent spectral features," Neural Networks, vol. 55, 2014.

[19] R. N. Khushaba, A. Al-Ani, A. Al-Timemy, and A. Al-Jumaily, "A fusion of time-domain descriptors for improved myoelectric hand control," in Computational Intelligence (SSCI), 2016 IEEE Symposium Series on. IEEE, 2016, pp. 1-6.

[20] M. Atzori, A. Gijsberts, C. Castellini, B. Caputo, A.-G. M. Hager, S. Elsig, G. Giatsidis, F. Bassetto, and H. Müller, "Electromyography data for non-invasive naturally-controlled robotic hand prostheses." Scientific data, vol. 1, p. 140053, 2014.

[21] A. Chan and G. Green, "Myoelectric control development toolbox," CMBES Proc., vol. 30, no. 1, 2007.

[22] A. Gijsberts and B. Caputo, "Exploiting accelerometers to improve movement classification for prosthetics," in IEEE International Conference on Rehabilitation Robotics (ICORR). IEEE, 2013, pp. 1-5. 